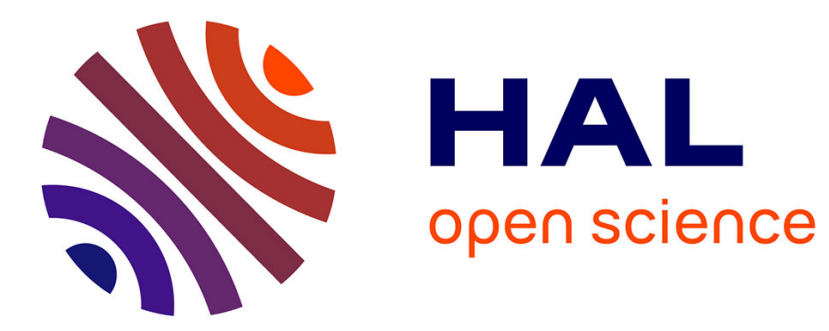

\title{
Optimization of strain selection in evolutionary continuous culture
}

Terence Bayen, Francis Mairet

\section{To cite this version:}

Terence Bayen, Francis Mairet. Optimization of strain selection in evolutionary continuous culture. International Journal of Control, 2016, 90 (12), pp.2748-2759. 10.1080/00207179.2016.1264631 . hal01332683

\section{HAL Id: hal-01332683 \\ https://hal.science/hal-01332683}

Submitted on 16 Jun 2016

HAL is a multi-disciplinary open access archive for the deposit and dissemination of scientific research documents, whether they are published or not. The documents may come from teaching and research institutions in France or abroad, or from public or private research centers.
L'archive ouverte pluridisciplinaire HAL, est destinée au dépôt et à la diffusion de documents scientifiques de niveau recherche, publiés ou non, émanant des établissements d'enseignement et de recherche français ou étrangers, des laboratoires publics ou privés. 


\title{
Optimization of strain selection in evolutionary continuous culture
}

\author{
Terence Bayen*, Francis Mairet ${ }^{\dagger}$
}

June 16, 2016

\begin{abstract}
In this work, we study a minimal time control problem for a perfectly mixed continuous culture with $n \geq 2$ species and one limiting resource. The model that we consider includes a mutation factor for the microorganisms. Our aim is to provide optimal feedback control laws to optimize the selection of the species of interest. Thanks to Pontryagin's Principle, we derive optimality conditions on optimal controls and introduce a sub-optimal control law based on a most rapid approach to a singular arc that depends on the initial condition. Using adaptive dynamics theory, we also study a simplified version of this model which allows to introduce a near optimal strategy.
\end{abstract}

Keywords. Process control. Biotechnology. Chemostat model. Optimal feedback. Pontryagin Maximum Principle. Singular control.

\section{Introduction}

Continuous culture can be used to select through competition microorganisms of interest from a pool of species (initially present or appearing through mutations) $[15,18]$. In a chemostat (i.e. with a constant dilution rate), the competitive exclusion principle (see [23]) states that the species which can grow at a rate equal to the dilution rate with the smallest substrate concentration survives whereas the others disappear as time goes to infinity. Thus, this operating mode allows species selection. The question, then, is whether it is possible to drive the competition between species in order to speed up the selection process. Based on optimal control theory, our aim is to find an optimal dilution strategy in order to minimize the time to reach a certain target where a given species is predominant.

This problem has been tackled in [1] in the case of two species. For this minimum time control problem, it is shown that the optimal strategy is of type Bang-Singular with at most one switching point, i.e. it is the concatenation of a bang arc (for which the control is extremal) and of a singular arc (for which the control is not extremal). The singular arc consists in the regulation of the substrate (i.e. an auxostat) at a set-point corresponding to the maximum of the difference between the growth rates of both species. Considering a more realistic framework of a selection process, we study in this paper the case of $n \geq 2$ species with mutations: each species $i$ gives rise to neighbor species $i-1$ and $i+1$. We use the Pontryagin Maximum Principle (see [22]) to derive necessary optimality conditions on optimal controls, and we show that any singular arc is contained in a hyperplane of the state space corresponding to a constant substrate concentration $\sigma$. This allows us to propose a feedback control law of type Bang-Singular as in the case $n=2$. We present numerical simulations obtained by a direct method that validate the proposed control law based on a most rapid approach to a singular arc (see $[1,7]$ ). For $n \geq 3$ and contrary to the case $n=2$, the singular arc may depend on the initial condition i.e. the value of $\sigma$ depends both on the characteristics of the system and on the initial condition. This phenomenon has been also encountered in [14] for the optimization of a fed-batch system. An alternative approach to the determination of $\sigma$ is to use adaptive dynamics (see $[10,11]$ ). This will allow us to provide a near optimal strategy that can be useful for a practitioner.

The paper is organized as follows. After the problem statement (Section 2), we apply in Section 3 the Pontryagin Maximum Principle to derive optimality conditions on the problem and we tackle the determination of an optimal value $\sigma$ for the singular arc using second order necessary conditions (see [4, 17]). We also provide

\footnotetext{
*Laboratoire IMAG, UMR CNRS 5149, Université Montpellier, CC 051, 34095 Montpellier cedex 5, France. tbayen@math. univ-montp2.fr

†Inria Biocore, BP93, 06902 Sophia-Antipolis Cedex, France. francis.mairet@inria.fr
} 
numerical simulations to validate the proposed control law based on a most rapid approach to a singular arc that depends on initial conditions. In the last section, we propose a near-optimal strategy for a simplified model derived from the adaptive dynamics theory which can be easily implemented and also overcomes the difficulty to obtain an optimal value of $\sigma$ along a singular arc.

\section{Statement of the problem}

We consider the growth of $n$ species (with concentration $x_{i}, 1 \leq i \leq n$ ) in competition for one limiting substrate (with concentration $s$ ) in a chemostat model (see [23]). Assuming small mutations with a constant mutation rate $a \ll 1$ (i.e. mutations of species $i$ creates species $i-1$ and $i+1$ ), the mutation-selection process can be represented by the following controlled system:

$$
\left\{\begin{array}{l}
\dot{x}_{i}=\left(\mu_{i}(s)-u\right) x_{i}+a\left(x_{i+1}+x_{i-1}\right)-2 a_{i} x_{i}, \\
\dot{s}=-\sum_{1 \leq j \leq n} \mu_{j}(s) x_{j}+u\left(s_{i n}-s\right),
\end{array}\right.
$$

where $1 \leq i \leq n$ and the convention $x_{0}=x_{n+1}=0$. The parameter $a_{i}$ is defined by:

$$
a_{i}:=\left\{\begin{array}{lll}
a / 2 & \text { if } & i \in\{1, n\} \\
a & \text { if } & i \in\{2, \ldots, n-1\}
\end{array}\right.
$$

Here, $s_{i n}$ is the input substrate concentration, $u$ is the dilution rate (which is the control variable in this setting), $\mu_{i}$ is the specific growth rate of species $i$, and we have considered adimensioned yield coefficients. System (2.1) can be seen as an approximation of the population dynamics model

$$
\begin{cases}\partial_{t} x(t, \varphi) & =(\mu(s(t), \varphi)-u) x(t, \varphi)+\alpha \Delta_{\varphi} x(t, \varphi), \\ \dot{s}(t) & =-\int_{\mathbb{R}_{+}^{*}} \mu(s(t), \varphi) x(t, \varphi) d \varphi+u\left(s_{i n}-s(t)\right),\end{cases}
$$

where $x(t, \varphi)$ stands for the distribution of a phenotypic trait $\varphi \in \mathbb{R}_{+}^{*}, \mu$ is the growth rate function depending on $s$ and $\varphi$, and the diffusion term $\Delta_{\varphi} x(t, \varphi)$ represents mutations (here $\alpha>0$ ). Such kind of model has been widely used in adaptive dynamics in order to represent and analyze evolution by selection and mutations (see e.g. $[19,21])$.

As a case study, we consider the following hypotheses on the specific growth rates (see Fig. 1):

(H1) The growth functions of the species for (2.1) are of Monod type:

$$
\mu_{i}(s):=\bar{\mu} \frac{s}{k_{i}+s}, \quad 1 \leq i \leq n,
$$

where $\bar{\mu}>0$ is the maximum growth rate and $0<k_{1}<k_{2}<\ldots<k_{n}$ are the half-saturation constants.

This hypothesis is based on experimental observations in chemostat cultures: the mutation-selection process can increase the number of substrate transporters and thus decrease the half-saturation constant $k_{i}$ (see $e . g$ $[13,20])$.

It is convenient to introduce the variable $M$ defined by $M:=s+\sum_{1 \leq j \leq n} x_{j}$ which represents the total mass of the system. By differentiating $M$ w.r.t. $t$, one finds:

$$
\dot{M}=u\left(s_{\text {in }}-M\right) \text {. }
$$

Therefore the set $E$ defined by

$$
E:=\left\{(x, s) \in \mathbb{R}_{+}^{*} \times \cdots \times \mathbb{R}_{+}^{*} \times\left[0, s_{i n}\right] ; s+\sum_{1 \leq j \leq n} x_{j}=s_{i n}\right\},
$$

is invariant and attractive for system (2.1). For sake of simplicity, we consider initial conditions in this set, so the system can be reduced as follows:

$$
\dot{x}_{i}=\left(\mu_{i}(s)-u\right) x_{i}+a\left(x_{i+1}+x_{i-1}\right)-2 a_{i} x_{i},
$$



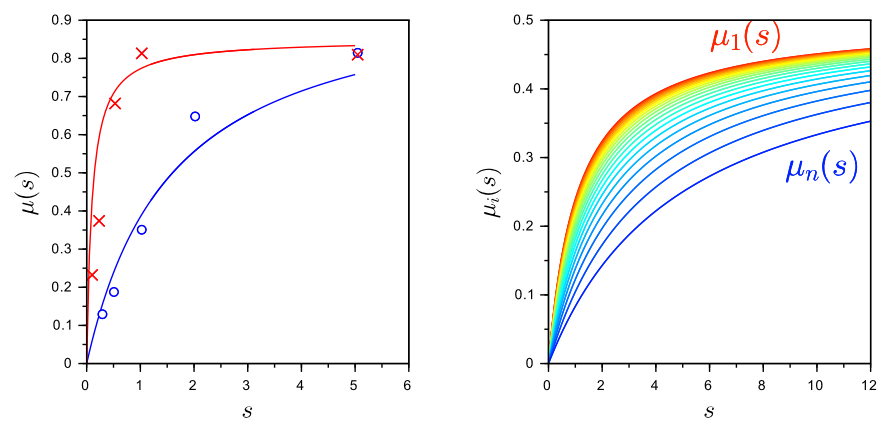

Figure 1: Picture Left: Specific growth rate for the initial strain (in blue) and the selected strain (in red) after ten days of chemostat culture [20]. Picture right: Specific growth rate $\mu_{i}$ for $n=20$ species.

where $1 \leq i \leq n$ and $s=s_{i n}-\sum_{1 \leq j \leq n} x_{j}$. Initial conditions for (2.6) are taken within the invariant set $F$ defined by

$$
F:=\left\{x \in \mathbb{R}_{+}^{n} ; 0<\sum_{1 \leq j \leq n} x_{j} \leq s_{i n}\right\} .
$$

Given $\eta \geq 0$ we also consider the following set of initial conditions $F_{\eta}$ defined by:

$$
F_{\eta}:=\left\{x \in F ; x_{1} \geq \eta \text { and } x_{i} \geq 0,2 \leq i \leq n\right\} .
$$

It will be useful when studying the system without mutations (i.e. when $a=0$ ). In this case, we will assume that the concentration of the first species is initially over a given threshold (but all the species do not need to be present initially). The set of admissible controls is defined by:

$$
\mathcal{U}:=\left\{u:[0,+\infty) \rightarrow\left[0, u_{\max }\right] ; u \text { meas. }\right\},
$$

where $u_{\max }$ denotes the maximal dilution rate. Without any loss of generality we suppose that $u_{\max }=1$. Next, we consider that the dilution rate can be chosen large enough in order to compete the growth of the species which amounts to do the following hypothesis:

(H2) The parameter $\bar{\mu}$ is such that $\bar{\mu}<1$.

The target set $\mathcal{T}$ is then defined by:

$$
\mathcal{T}:=\left\{x \in F ; \sum_{1 \leq j \leq n} k_{j} x_{j} \leq \tilde{k} \sum_{1 \leq j \leq n} x_{j}\right\},
$$

where $k_{1}<\cdots<k_{p}<\tilde{k}<k_{p+1}<\cdots<k_{n}$, with $p \in\{2, \ldots, n-2\}$. It corresponds to the selection of a population with a low weighted averaged half-saturation constant $\hat{k}$ :

$$
\hat{k}:=\frac{\sum_{1 \leq j \leq n} k_{j} x_{j}}{\sum_{1 \leq j \leq n} x_{j}} \leq \tilde{k} .
$$

The optimal control problem can be now stated as follows. Given an initial condition $x^{0}=\left(x_{1}^{0}, \ldots, x_{n}^{0}\right) \in F$, our aim is to find a control $u \in \mathcal{U}$ steering the solution $x_{u}(\cdot)$ of (2.6) from $x^{0}$ to the target $\mathcal{T}$ in minimal time:

$$
\inf _{u \in \mathcal{U}} T_{u} \quad \text { s.t. } \quad x_{u}\left(T_{u}\right) \in \mathcal{T},
$$

where $T_{u} \in[0,+\infty]$ is the first entry time into the target $\mathcal{T}$. If possible, one would like to obtain a feedback control law driving (2.6) in minimal time to the target $\mathcal{T}$.

Let us now discuss the existence of an optimal control for (2.9). Without mutation (i.e. when $a=0$ ), the competitive exclusion principle (see [23]) implies that for a constant value for the control $u<\mu_{1}\left(s_{i n}\right)$, we have:

$$
\lim _{t \rightarrow+\infty} x_{1}(t)>0 \quad \text { and } \quad \lim _{t \rightarrow+\infty} x_{i}(t)=0, \quad i>1 .
$$


This property then provides a strategy that steers any initial condition to the target set in finite horizon (as $\left.k_{1}<\tilde{k}\right)$. With mutations, it has been shown in [9] that the washout is the unique steady state on the boundary of $\mathbb{R}_{+}^{n}$, i.e. there are no mono-species steady-states. Nonetheless, in the limit of small mutations, it was proved in [19] that the population converges towards dirac masses on a structured model of species competition. In our case study, we give the following controllability result.

Proposition 2.1. Given $\eta>0$, there exists $a_{0}>0$ such that for any mutation rate a satisfying $0 \leq a \leq a_{0}$, the target is reachable from any initial condition in $F_{\eta}$.

Proof. Let $\varepsilon>0$ be such that $\tilde{k}-\varepsilon>k_{1}$ and $\mathcal{T}_{\varepsilon} \subset \mathcal{T}$ be defined by:

$$
\mathcal{T}_{\varepsilon}:=\left\{x \in F ; \sum_{1 \leq j \leq n} k_{j} x_{j} \leq(\tilde{k}-\varepsilon) \sum_{1 \leq j \leq n} x_{j}\right\}
$$

First step: study of the case $a=0$. For any initial condition $x^{0} \in F_{\eta}$, any constant control $\bar{u}<\mu_{1}\left(s_{i n}\right)$ steers the unique solution of (2.6) with $a=0$ from $x^{0}$ to the set $\mathcal{T}_{\varepsilon}$ in a finite time $\tau_{\bar{u}}\left(x^{0}\right)$. This follows using the competitive exclusion principle as $k_{1}<\tilde{k}-\varepsilon$ (see [23] and recall (2.10)). Moreover, for any initial condition in $F_{\eta}$, we can utilize the same value of the control (i.e. $u=\bar{u}$ ). Now, the mapping $x^{0} \mapsto \tau_{\bar{u}}\left(x^{0}\right)$ can be interpreted as the first entry time into the target $\mathcal{T}_{\varepsilon}$. Therefore it corresponds to the minimal time function to reach $\mathcal{T}_{\varepsilon}$ from $x^{0}$ with $(2.6)$ and where the set of admissible controls is reduced to the constant control equal to $\bar{u}$. Using standard regularity results for the value function (see [6]), the mapping $\tau$ is continuous over $F_{\eta}$ which is a compact subset of $\mathbb{R}_{+}^{n}$. Hence, $\tau$ is bounded over this set and thus there exists $T>0$ such that for any $x^{0} \in F_{\eta}$, the solution of (2.6) with $a=0$ reaches $\mathcal{T}_{\varepsilon}$ in time less or equal than $T$.

Second step: study of the case $a \neq 0$. The dynamics (2.6) depends linearly on the parameter $a \geq 0$. By classical results of differentiable dependance w.r.t. parameters for the solutions of an ordinary differential equation, the mapping $a \longmapsto x\left(T, x^{0}, a\right)$ is of class $C^{1}$ (where $x\left(\cdot, x^{0}, a\right)$ denotes the unique solution of (2.6) starting from $x^{0}$ at $\left.t=0\right)$. We deduce that for $1 \leq i \leq n$, one has:

$$
\frac{\partial x_{i}}{\partial a}\left(T, x_{0}, a\right)=\int_{0}^{T} g_{i}\left(x\left(t, x^{0}, a\right)\right) \mathrm{d} t
$$

where $g_{i}(x):=x_{i+1}+x_{i-1}-2 x_{i}$ for $2 \leq i \leq n, g_{1}(x)=x_{2}-x_{1}$, and $g_{n}(x)=x_{n-1}-x_{n}$. As trajectories lie in the set $F$, one has $0 \leq x_{i}(t) \leq s_{i n}$ for any time $t \geq 0$ and for $1 \leq i \leq n$. Thus, one finds:

$$
\left|x_{i}\left(T, x^{0}, a\right)-x_{i}\left(T, x^{0}, 0\right)\right| \leq \sup _{a \geq 0}\left|\int_{0}^{T} g_{i}\left(x\left(t, x^{0}, a\right)\right) \mathrm{d} t\right| \leq 4 T s_{i n}|a|, \quad 1 \leq i \leq n .
$$

In particular, there exists $a_{0}>0$ such that for any $a \in \mathbb{R}$, one has:

$$
0 \leq a \leq a_{0} \quad \Rightarrow \quad \forall x^{0} \in F_{\eta}, \quad x\left(T, x^{0}, a\right) \in \mathcal{T}_{\frac{\varepsilon}{2}} .
$$

It follows that for any $a \in\left[0, a_{0}\right]$, any solution of (2.6) starting in $F_{\eta}$ has reached the target set $\mathcal{T}$ in time less than $T$ which ends the proof.

Remark 2.1. (i) We point out the fact that if $a=0$ and $x_{i}(0)=0$ for $1 \leq i \leq p$, then the target cannot be reached by (2.6) with $a=0$. Thus, the reasoning above does not hold any more. This explains the choice of taking initial conditions in the set $F_{\eta}$.

(ii) If now $x_{1}^{0}>0$ but $x^{0} \notin F_{\eta}$, then the previous proof shows that the system can reach the target in a finite horizon $T \geq 0$. However, $T$ could be arbitrarily long in this case as $x_{1}^{0}$ can be very small. Nevertheless, it is still possible to apply the reasoning above to show that for a fixed initial condition $x^{0} \in F$ such that $x_{1}^{0}>0$, then if a is small enough the system (2.6) will reach the target in a finite horizon. In that case, a $a_{0}$ is not uniform (i.e. it depends on initial conditions).

We now investigate optimal strategies steering (2.6) in minimal time to the target set $\mathcal{T}$. 


\section{Optimality results}

\subsection{Pontryagin Maximum Principle}

In this part, we derive necessary optimality conditions for problem (2.9) using the Pontryagin Maximum Principle (PMP), see e.g [22]. First, the existence of an optimal control for problem (2.9) follows directly from Fillipov's Theorem (see e.g [8]) using standard compactness arguments and the fact that the target can be reached from any initial condition in $F_{\eta}(\eta>0)$ (this is guaranteed by Proposition 2.1 at least when $a$ is small enough). Recall now that the normal cone $N_{C}(x)$ to a non-empty closed convex set $C \subset \mathbb{R}^{n}$ is defined at some point $x \in C$ by $N_{C}(x):=\left\{y \in \mathbb{R}^{n},\langle y, c-x\rangle \leq 0, \forall c \in C\right\}$ where $\langle\cdot, \cdot\rangle$ denotes the usual scalar product over $\mathbb{R}^{n}$. We denote by $H: \mathbb{R}^{n} \times \mathbb{R}^{n} \times \mathbb{R} \times \mathbb{R} \rightarrow \mathbb{R}$ the Hamiltonian associated to (2.9), that is:

$$
H=H(x, \lambda, \eta, u)=\sum_{1 \leq j \leq n} \lambda_{j}\left(\mu_{j}(s) x_{j}+a\left(x_{j-1}+x_{j+1}\right)-2 a_{j} x_{j}\right)-u \sum_{1 \leq j \leq n} \lambda_{j} x_{j}+\eta,
$$

where $\lambda=\left(\lambda_{1}, \cdots, \lambda_{n}\right)$ denotes the adjoint vector. Let $u \in \mathcal{U}$ an optimal control, $x(\cdot)$ the corresponding trajectory starting from a point $x^{0} \in F$, and $T_{u}$ the terminal time corresponding to the control $u$. Then, there exists an absolutely continuous function $\lambda:\left[0, T_{u}\right] \rightarrow \mathbb{R}^{n}$ and $\eta \leq 0$ such that the following conditions are satisfied:

- The pair $(\lambda, \eta)$ is non-trivial i.e.:

$$
(\lambda(\cdot), \eta) \neq 0 .
$$

- With the convention that $\lambda_{0}=\lambda_{n+1}=0$, the adjoint vector satisfies almost everywhere the adjoint equation on $\left[0, T_{u}\right]: \dot{\lambda}_{i}(t)=-\frac{\partial H}{\partial x_{i}}(x(t), \lambda(t), \eta, u(t)), 1 \leq i \leq n$, or equivalently

$$
\dot{\lambda}_{i}=-\lambda_{i}\left(\mu_{i}(s)-u\right)-a\left(\lambda_{i-1}+\lambda_{i+1}\right)+2 a_{i} \lambda_{i}+\sum_{1 \leq j \leq n} \lambda_{j} \mu_{j}^{\prime}(s) x_{j}, \quad 1 \leq i \leq n .
$$

- The control $u$ satisfies the maximization condition almost everywhere on $\left[0, T_{u}\right]$ :

$$
u(t) \in \arg \max _{0 \leq v \leq 1} H(x(t), \lambda(t), \eta, v) .
$$

- The adjoint vector satisfies the transversality condition at the terminal time $T_{u}$ (see e.g. [24]):

$$
\lambda\left(T_{u}\right) \in-N_{\mathcal{T}}\left(x\left(T_{u}\right)\right) .
$$

We call extremal trajectory a quadruplet $(x, \lambda, \eta, u)$ satisfying (2.6)-(3.2)-(3.3). If $\eta<0$, resp. $\eta=0$, then we say that the extremal is a normal extremal, resp. an abnormal extremal. In the normal case, we can always assume that $\eta=-1$ by homogeneity of the Hamiltonian and the linearity of (3.2). As the system is autonomous, the Hamiltonian is conserved along any extremal trajectory, moreover the value of $H$ is zero as the terminal time is free. Hence, any extremal trajectory satisfies:

$$
H(x(t), \lambda(t), \eta, u(t))=0 .
$$

for any time $t \in\left[0, T_{u}\right]$. The switching function $\phi$ associated to the control $u$ is defined by:

$$
\phi:=\frac{\partial H}{\partial u}=-\sum_{1 \leq j \leq n} \lambda_{j} x_{j}
$$

From (3.3), we obtain the following control law. For a.e. $t \in\left[0, T_{u}\right]$ one has:

$$
\left\{\begin{array}{l}
\phi(t)>0 \quad \Rightarrow \quad u(t)=1, \\
\phi(t)<0 \quad \Rightarrow \quad u(t)=0, \\
\phi(t)=0 \quad \Rightarrow \quad u(t) \in[0,1] .
\end{array}\right.
$$

If the control $u$ is non-constant in any neighborhood of a point $t_{0}$, then we say that $t_{0}$ is a switching point which implies that $\phi\left(t_{0}\right)=0$. A singular arc is a time interval $I=\left[t_{1}, t_{2}\right]$ where the switching function $\phi$ 
vanishes over $I$. We then say that the trajectory is singular on the interval $I$ (see $[4,17]$ ). The singular control is defined as the control $u_{s}(\cdot)$ such that the associated trajectory is singular (its expression can be obtained by differentiating twice $\phi$ w.r.t. $t$ in the case of a singular arc of order 1 , see $[4,17])$. We say that the singular arc is admissible provided that $u_{s} \in[0,1]$ over $I$. If the switching function is such that $\phi>0$, resp. $\phi<0$ on a time interval $I$, then we say that the trajectory contains a bang arc $B_{+}$, resp. a bang arc $B_{-}$. The behavior of $\phi$ is important to study the optimal trajectories. By differentiating the switching function w.r.t. $t$, we find that $\dot{\phi}$ satisfies:

$$
\dot{\phi}=-\left(s_{i n}-s\right) \sum_{1 \leq j \leq n} \lambda_{j} \mu_{j}^{\prime}(s) x_{j}
$$

over the interval $\left[0, T_{u}\right]$. Let us now explicit the transversality condition. As the boundary of $\mathcal{T}$ is a hyperplane, the adjoint vector at the terminal time is given by

$$
\lambda\left(T_{u}\right)=-\alpha\left[\begin{array}{c}
k_{1}-\tilde{k} \\
\vdots \\
k_{n}-\tilde{k}
\end{array}\right],
$$

where $\alpha \in \mathbb{R}_{+}$. Using that $x\left(T_{u}\right)$ belongs to the boundary of the set $\mathcal{T}$, we find that

$$
\phi\left(T_{u}\right)=0
$$

Hence, the switching function vanishes at the first entry time into the target. As a consequence, it can be expected that an optimal trajectory is singular in a left neighborhood of the terminal time $T_{u}$ in order to satisfy the transversality condition (3.10).

\subsection{Characterization of the singular arcs}

Singular arcs are of particular interest in the optimal synthesis of the problem. In fact, we will see that the singular control can be expressed in feedback form (that is, $u_{s}$ will be written in terms of the state variables only). Moreover, they provide a feeding strategy steering (2.6) to the target set. In the next proposition, we give a geometric characterization of singular arcs.

Proposition 3.1. Suppose that an extremal trajectory contains a singular arc on a time interval $I:=\left[t_{1}, t_{2}\right]$. Then, there exists $\sigma \in\left(0, s_{i n}\right)$ such that for any time $t \in I$ we have $s(t)=\sigma$.

Proof. Consider an extremal trajectory defined over a time interval $\left[0, T_{u}\right]$ and suppose that there exists a time interval $I=\left[t_{1}, t_{2}\right]$ where $\phi$ is zero. Then, by differentiating $\phi$ w.r.t. $t$, we obtain:

$$
\sum_{1 \leq j \leq n} \lambda_{j} x_{j}(t) \mu_{j}^{\prime}(s(t))=0,
$$

for any time $t \in I$. Suppose now that there exists $t_{0}$ in the interior of $I$ such that $\dot{s}\left(t_{0}\right) \neq 0$. By standard results in optimal control theory (see e.g [5]) we know that a singular control is smooth in a neighborhood of $t_{0}$ in $I$. Thus, $\dot{s}$ is continuous in a neighborhood $\mathcal{V}$ of $t_{0}$ and we deduce that $\dot{s}(t) \neq 0$ for $t \in \mathcal{V}$. By differentiating (3.11) w.r.t. $t$ and using the fact that $\dot{s} \neq 0$ in $\mathcal{V}$, we find that

$$
\sum_{1 \leq j \leq n} \lambda_{j}(t) x_{j}(t) \mu_{j}^{\prime \prime}(s(t))=0 .
$$

By induction, it follows that for any $t \in \mathcal{V}$ we have:

$$
\sum_{1 \leq j \leq n} \lambda_{j}(t) x_{j}(t) \mu_{j}^{(k)}(s(t))=0, \quad 1 \leq k \leq n,
$$

where $s \longmapsto \mu_{j}^{(k)}(s)$ is the $k$-th derivative of $\mu_{j}$ w.r.t. $s$. Now, let us define a map $y: \mathcal{V} \rightarrow \mathbb{R}^{n}$ by:

$$
y(t):=\left(\lambda_{1}(t) x_{1}(t), \ldots, \lambda_{n}(t) x_{n}(t)\right),
$$

and a matrix $A(s)=\left(a_{k, j}(s)\right)_{1 \leq k, j \leq n} \in \mathcal{M}_{n}(\mathbb{R})$ by $a_{k, j}(s):=\mu_{j}^{(k)}(s)$. From (3.13), we find that for any time $t \in \mathcal{V}, y(t)$ is in the kernel of $\bar{A}(s(t))$. Now, as the $\mu_{j}$ are rational fractions linearly independent (see (H1)), 
the equation $\operatorname{det}(A(s))=0$ has at most a finite number of solutions over $\mathbb{R}$. If $Z$ denotes the set of solutions of the algebraic equation $\operatorname{det}(A(s))=0$, then by reducing $\mathcal{V}$ if necessary, we can find a neighborhood $\mathcal{V}^{\prime}$ of $t_{0}$ where $y(t) \in \operatorname{ker} A(s(t))$ and $s(t) \notin Z$ for any time $t \in \mathcal{V}^{\prime}$. Therefore, the vector $y(t)$ must be zero in $\mathcal{V}^{\prime} i . e$. we must have $\lambda=0$ on $\mathcal{V}^{\prime}$. As the adjoint equation is linear w.r.t. $\lambda$, we obtain that $\lambda$ is zero along [0,Tu . If $\eta$ is non-zero, then we obtain a contradiction with $H=0$. We deduce that $\eta$ must be zero and we have again a contradiction with the PMP as we would have $(\eta, \lambda)=0$. Thus one has $\dot{s}(t)=0$ for every time $t \in I$. As $t \longmapsto u_{s}(t)$ is smooth over $I$, we deduce that $s$ is constant over $I$ which ends the proof.

Hereafter, we will denote by $S_{\sigma}$ a singular arc corresponding to a constant substrate concentration $s=\sigma$ over a time interval $I$, which corresponds to an auxostat (see e.g [2]). In the invariant manifold $F$, a singular arc corresponds also to a turbidostat (i.e. a constant biomass concentration). These operating modes are commonly used for strain selection (see e.g [2]). We now show that a singular arc is always admissible:

Corollary 3.1. Consider a singular extremal trajectory defined over a time interval $I:=\left[t_{1}, t_{2}\right]$ and let $\sigma \in\left(0, s_{i n}\right)$ such that for any $t \in I$ one has $s(t)=\sigma$. Then, the singular control $u_{s}$ is admissible and can be expressed in feedback form as:

$$
u_{s}[x]=\frac{\sum_{1 \leq j \leq n} \mu_{j}(\sigma) x_{j}}{\sum_{1 \leq j \leq n} x_{j}} .
$$

Proof. As we have $\dot{s}=0$ along a singular arc, we obtain (3.14) from (2.6). From (H2), we have $\mu_{j}(s)<1$, $1 \leq j \leq n$ for any $s \in\left[0, s_{i n}\right]$. Thus, we deduce that $0 \leq u_{s}[x] \leq 1$ which concludes the proof.

If in addition a singular extremal trajectory reaches the target, then the following properties hold.

Proposition 3.2. Consider an optimal trajectory which is singular over a time interval of type $\left[t_{1}, T_{u}\right]$ with $t_{1}<T_{u}$. Then there exists $\sigma \in\left[0, s_{i n}\right]$ such that:

$$
\sum_{1 \leq j \leq n}\left(k_{j}-\tilde{k}\right) x_{j}\left(T_{u}\right) \mu_{j}^{\prime}(\sigma)=0
$$

and

$$
\sum_{1 \leq j \leq n}\left(k_{j}-\tilde{k}\right) x_{j}\left(T_{u}\right) \mu_{j}^{\prime \prime}(\sigma) \geq 0 .
$$

Proof. We obtain (3.15) combining (3.9) and (3.11). Now, by differentiating $\phi$ twice w.r.t. $t$, one obtains:

$$
\ddot{\phi}=-\dot{s}\left(s_{i n}-s\right) \sum_{1 \leq j \leq n} \lambda_{j} \mu_{j}^{\prime \prime}(\sigma) x_{j}+\frac{1}{s_{i n}-s} \dot{\phi}\left(\dot{s}-\sum_{1 \leq j \leq n} \mu_{j}^{\prime}(s) x_{j}\right) .
$$

Therefore, we find that for $t \in\left[t_{1}, T_{u}\right]$ one has:

$$
\frac{\partial}{\partial u} \frac{d^{2} H_{u}}{d t^{2}}(x(t), \lambda(t), \eta, u(t))=-\left(s_{i n}-s(t)\right)^{2} \sum_{1 \leq j \leq n} \lambda_{j}(t) \mu_{j}^{\prime \prime}(\sigma) x_{j}(t)
$$

We know that if a singular arc is optimal, then it satisfies the so-called Legendre-Clebsch condition (see e.g $[4,17])$ which amounts to saying that the following inequality holds:

$$
\frac{\partial}{\partial u} \frac{d^{2} H_{u}}{d t^{2}}(x(t), \lambda(t), \eta, u(t)) \geq 0 \quad \forall t \in\left[t_{1}, t_{2}\right] .
$$

We thus obtain (3.16) using (3.9) which concludes the proof.

A deeper insight into the characterization of a singular arc is given by the next proposition which provides a range for $\sigma$.

Proposition 3.3. Consider an optimal trajectory which is singular over a time interval of type $\left[t_{1}, T_{u}\right]$ with $t_{1}<T_{u}$. Then, the value of $\sigma$ along the singular arc satisfies $\sigma \in\left(k_{1}, k_{n}\right)$. 
Proof. First, note that for $1 \leq i \leq n$, one has:

$$
\frac{\partial \nu_{i}}{\partial k_{i}}=\bar{\mu} \frac{s-k_{i}}{\left(s+k_{i}\right)^{3}}
$$

where $\nu_{i}\left(k_{i}, s\right):=\mu_{i}^{\prime}(s)=\frac{\bar{\mu} k_{i}}{\left(k_{i}+s\right)^{2}}$ depends both on $s \in\left[0, s_{i n}\right]$ and $k_{i} \in \mathbb{R}_{+}^{*}$. Now, suppose that $\sigma \leq k_{1}$. It follows that $\frac{\partial \nu_{i}}{\partial k_{i}}(k, \sigma)<0$ for $k \in\left(k_{1}, k_{n}\right]$, and so we get:

$$
\mu_{1}^{\prime}(\sigma)>\mu_{2}^{\prime}(\sigma)>\ldots>\mu_{n}^{\prime}(\sigma)>0
$$

At the terminal time $T_{u}$, the trajectory reaches the target and thus, the transversality condition implies:

$$
\sum_{1 \leq j \leq p}\left(\tilde{k}-k_{j}\right) x_{j}\left(T_{u}\right)=\sum_{p+1 \leq j \leq n}\left(k_{j}-\tilde{k}\right) x_{j}\left(T_{u}\right)>0 .
$$

Using the previous inequality and (3.17), one finds:

$$
\begin{aligned}
\sum_{1 \leq j \leq p}\left(\tilde{k}-k_{j}\right) x_{j}\left(T_{u}\right) \mu_{j}^{\prime}(\sigma)>\mu_{p}^{\prime}(\sigma) \sum_{1 \leq j \leq p}\left(\tilde{k}-k_{j}\right) x_{j}\left(T_{u}\right) & =\mu_{p}^{\prime}(\sigma) \sum_{p+1 \leq j \leq n}\left(k_{j}-\tilde{k}\right) x_{j}\left(T_{u}\right) \\
& >\sum_{p+1 \leq j \leq n}\left(k_{j}-\tilde{k}\right) x_{j}\left(T_{u}\right) \mu_{j}^{\prime}(\sigma) .
\end{aligned}
$$

Finally, we get the inequality $\sum_{1 \leq j \leq n}\left(k_{j}-\tilde{k}\right) x_{j}\left(T_{u}\right) \mu_{j}^{\prime}(\sigma)<0$, in contradiction with (3.15), so we must have $\sigma>k_{1}$. Similarly, we can show that $\sigma<k_{n}$.

It is also worth noting that any singular trajectory drives the system to the target (provided that $a$ is small enough) as shown in the next proposition.

Proposition 3.4. Given $\eta>0$, there exists $a_{0}>0$ such that for any $a \in\left[0, a_{0}\right]$, then for any value of $\sigma \in\left(0, s_{i n}\right)$, the singular trajectory $S_{\sigma}$ drives (2.6) from any initial condition in $F_{\eta}$ into the target in finite horizon.

Proof. For $1 \leq i \leq n$, let $p_{i}:=\frac{x_{i}}{s_{i n}-s}$ the fraction of species $i$ in the system (recall that we have $s_{i n}-s=$ $\left.\sum_{j=1}^{n} x_{j}\right)$. By differentiating $p_{i}$ w.r.t. $t$, we get:

$$
\dot{p}_{i}=p_{i} \sum_{1 \leq j \leq n}\left(\mu_{i}(s)-\mu_{j}(s)\right) p_{j}+a\left(p_{i+1}+p_{i-1}\right)-2 a_{i} p_{i}, \quad 1 \leq i \leq n
$$

with the convention that $p_{0}=p_{n+1}=0$. We now consider a singular arc defined over a time interval $\left[t_{1}, t_{2}\right]$, and let $\sigma \in\left(0, s_{i n}\right)$ be such that $s(t)=\sigma$ for any time $t \in\left[t_{1}, t_{2}\right]$.

First step: we suppose that $a=0$. From (3.18), the mapping $t \longmapsto p_{1}(t)$ is increasing and bounded by 1 . Thus, there exists $p_{1}^{\infty} \in(0,1]$ such that $p_{1}(t) \rightarrow p_{1}^{\infty}$ when $t \rightarrow+\infty$. Now, let us set $\alpha_{i}:=\sum_{1 \leq j \leq n}\left|\mu_{i}(\sigma)-\mu_{j}(\sigma)\right|>0$ and $\alpha:=\max _{1 \leq i \leq n}\left(\alpha_{i}\right)$. As one has $0 \leq p_{i} \leq 1$ for $1 \leq i \leq n$, we deduce that

$$
0 \leq\left|\dot{p}_{i}\right| \leq \alpha_{i} \leq \alpha, \quad 1 \leq i \leq n
$$

and thus for $1 \leq i \leq n, p_{i}$ is uniformly continuous over $\mathbb{R}_{+}$. If we set $f(t):=\ln \left(p_{1}(t)\right)$, we deduce that $f(t)$ converges to a finite value when the time $t$ goes to infinity. Moreover, one has

$$
\dot{f}(t)=\sum_{j=2}^{n}\left(\mu_{1}(\sigma)-\mu_{j}(\sigma)\right) p_{j}(t), \quad t \geq 0
$$

and we deduce that $\dot{f}$ is uniformly continuous. Applying Barbalat's Lemma yields that $\dot{f}(t)$ converges to zero when $t$ got to infinity. Thus:

$$
\lim _{t \rightarrow+\infty} \sum_{j=2}^{n}\left(\mu_{1}(\sigma)-\mu_{j}(\sigma)\right) p_{j}(t)=0
$$


This implies that $p_{j}(t) \rightarrow 0$ when $t \rightarrow+\infty$ (using that $\mu_{1}(\sigma)-\mu_{j}(\sigma)>0$ for $j \geq 2$ ), and thus $p_{1}(t) \rightarrow 1$ when $t \rightarrow+\infty$. Finally, the quantity $\sum_{1 \leq j \leq n} k_{j} p_{j}$ converges to $k_{1}<\tilde{k}$. We have thus proved that given $\varepsilon>0$, any solution of (3.18) with $a=0$ enters the set $\mathcal{T}_{\varepsilon}$ (recall (2.11)) in a finite horizon. Using similar arguments as in the proof of Proposition 2.1, we infer that there exists $T>0$ such that any trajectory starting in the set $F_{\eta}$ will reach $\mathcal{T}_{\varepsilon}$ in time less than $T$.

Second step: we suppose that $a>0$. We utilize the differentiable dependency of the solutions of an ordinary differential equation w.r.t. parameters (see also the proof of Proposition 2.1). As (3.18) is linear w.r.t. $a$ and $0 \leq p_{i} \leq 1$, we deduce that there exists a constant $C>0$ such that for any $p^{0} \in[0,1]^{n}$ with $\sum_{1 \leq j \leq n} p_{j}^{0}=1$ one has:

$$
\left|p_{i}\left(T, p^{0}, a\right)-p_{i}\left(T, p^{0}, 0\right)\right| \leq C a, \quad 1 \leq i \leq n,
$$

where $t \longmapsto p_{i}\left(t, p^{0}, a\right)$ denotes the unique solution of (3.18) over $\mathbb{R}_{+}$starting from $p^{0}$ at time 0 . We can now conclude as in the proof of Proposition 2.1 to show that if $a$ is small enough, any solution of (3.18) starting from $F_{\eta}$ will enter the set $\mathcal{T}_{\frac{\varepsilon}{2}}$ in time less than $T$. This concludes the proof.

Remark 3.1. Similarly as in Remark 2.1 (ii), we can show that given an initial condition $x^{0} \notin F_{\alpha}$ such that $x_{1}^{0}>0$, there exists $a_{0}$ such that if $0 \leq a \leq a_{0}$, then the solution of (2.6) (corresponding to $S_{\sigma}$ ) will reach the target set in finite horizon (however, $a_{0}$ may not be uniform and depends on the initial condition $x^{0}$ ).

\subsection{Optimal synthesis and numerical simulations}

We provide in this section numerical simulations of (2.9) based on a direct method using the software bocop [3]. Together with section 3.2, this will allow us to propose a feedback control law driving optimally the system to the target set.

The direct method uses a time discretization to transform the infinite-dimensional optimal control problem into a nonlinear optimization problem, solved here by interior point techniques. Software bocop typically uses a discretization by a Lobatto IIIC formula (6th order), a constant initialization, and a tolerance for NLP solver set at $10^{-10}$. Numerical simulations have been performed with $n=10$ species, using 300 time steps. Fig. 2 depicts optimal trajectories, which are of the form Bang-Singular $B_{ \pm} S_{\sigma}$ i.e. a concatenation of a bang arc $u=0$ or $u=1$ (depending on the initial substrate concentration), and of a singular arc until reaching the target. We have repeated this simulation for different initial conditions. From Lemma 3.3 we know that $\sigma$ belongs to the interval $\left(k_{1}, k_{n}\right)$, which is verified numerically. On Fig. 3, we can see that the value of $\sigma$ along a singular arc depends on initial conditions (this is in line with the results of [14] when the dimension of the state space is greater than 3 ).

In view of the characterization of the singular arcs, we introduce a feedback control law $u_{\sigma}[\cdot]$ depending on the state $x=\left(x_{1}, \ldots, x_{n}\right)$ and a substrate concentration $\sigma$ as follows:

$$
\left\{\begin{array}{lll}
u_{\sigma}[x]=0 & \text { if } & \sum_{1 \leq j \leq n} x_{j}<s_{i n}-\sigma, \\
u_{\sigma}[x]=1 & \text { if } & \sum_{1 \leq j \leq n} x_{j}>s_{i n}-\sigma, \\
u_{\sigma}[x]=u_{s}[x] & \text { if } & \sum_{1 \leq j \leq n} x_{j}=s_{i n}-\sigma .
\end{array}\right.
$$

Based on the numerical simulations (see Fig. 2 and 3 ) and on section 3.2, we conjecture the following optimality result.

Conjecture 3.1. For any initial condition $x^{0} \in F$, there exists $\sigma\left(x^{0}\right) \in\left(k_{1}, k_{n}\right)$ such that the feedback control $u_{\sigma\left(x^{0}\right)}$ is optimal for $(2.9)$.

In other words, any optimal control is of type $B_{ \pm} S_{\sigma\left(x^{0}\right)}$. This control law can be interpreted as a most rapid approach (see e.g [7]) to a singular arc $s=\sigma\left(x^{0}\right)$ (depending on the initial condition $x^{0}$ ) : whenever $s>\sigma\left(x^{0}\right)$ or equivalently $\sum_{1 \leq j \leq n} x_{j}>s_{i n}-\sigma\left(x^{0}\right)$, then the control $u=0$ allows $s$ to decrease until reaching the value $\sigma\left(x^{0}\right)$. The same remark holds whenever the substrate concentration is such that $s<\sigma\left(x^{0}\right)$ using $u=1$.

We can also point out the fact that at time 0 , then $x_{1}$ is usually small (typical initial conditions for a monoclonal population are such that $x_{i}(0)=0$ for $\left.1 \leq i \leq n-1\right)$ whereas if the time goes to infinity, then $x_{1}$ will be positive and $x_{i} \ll 1$ for $i>1$. Hence, it is not evident how one can estimate the value of $\sigma\left(x^{0}\right)$. As its determination remains a difficult question, we now propose an alternative approach to (2.9). 

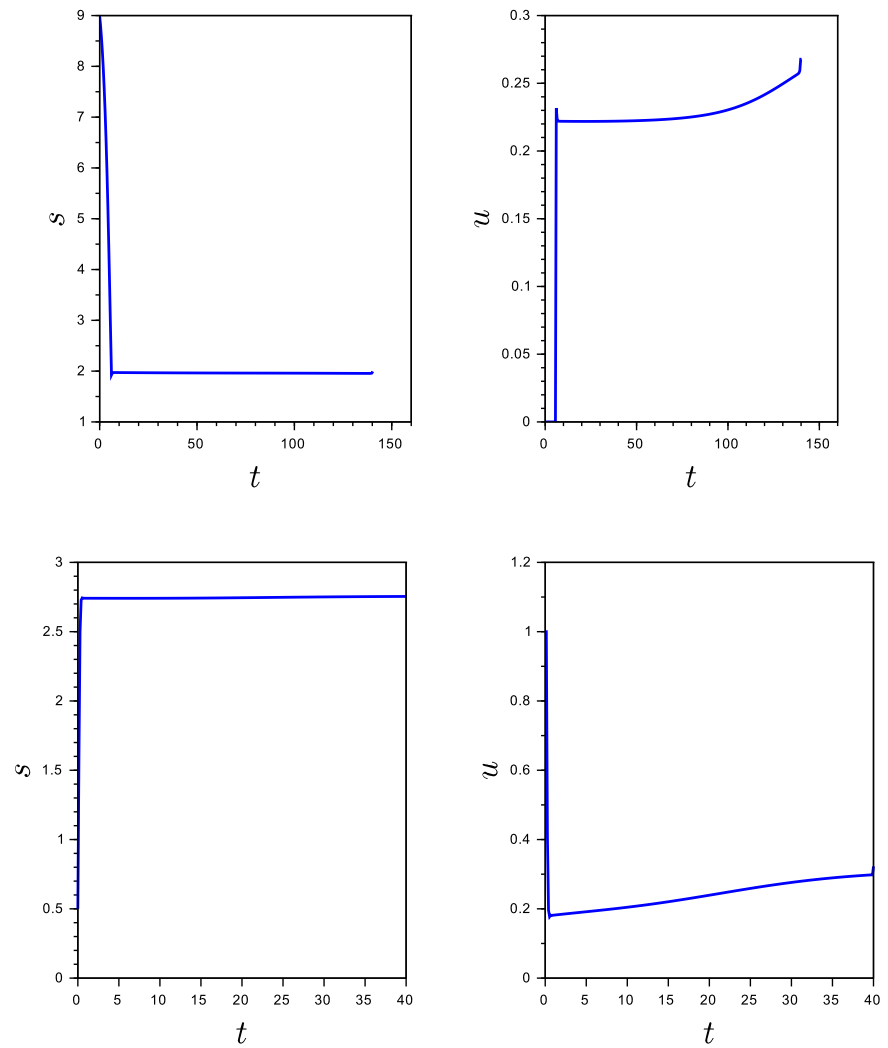

Figure 2: Optimal trajectories of type bang-singular obtained numerically by a direct method with $n=10$. Left: substrate concentration. Right: control $u$. Top: substrate concentration at $t=0$ is high, the optimal trajectory is of type $B_{-} S_{\sigma}$. Down: substrate concentration at $t=0$ is low, the optimal trajectory is of type $B_{+} S_{\sigma}$.

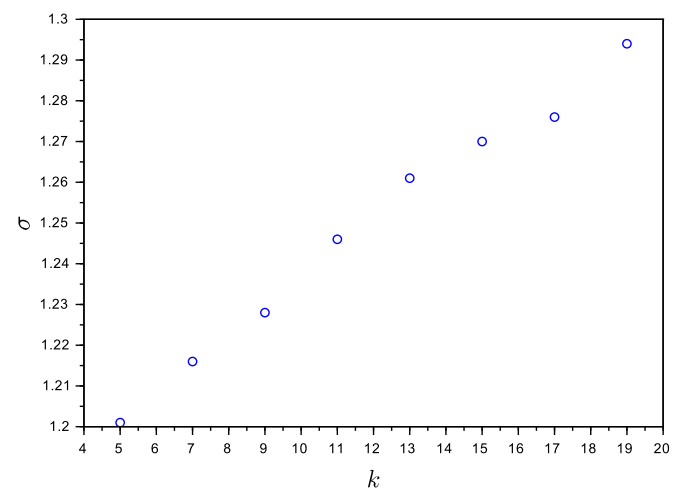

Figure 3: Effect of the initial condition on the value of $\sigma$ along a singular arc $S_{\sigma}$. The initial condition corresponds to a monoclonal population of species $k$ i.e. $x_{k}(0)=3$ and $x_{i}(0)=0$ for $i \neq k$. 


\section{Near optimal strategy}

In an evolutionary experiment, a chemostat system is inoculated with a species $x_{n}$, and the culture is operated in continuous mode in order to obtain new mutants. Nonetheless, it is not possible to guess how much the species will evolve (how much the half-saturation constant $k$ will decrease). Since the system is actually unknown, the value of $\sigma$ cannot be determined (even numerically). Here, we propose a near-optimal strategy, based on a model simplification assuming very small mutation rate, that can be implemented easily. The methodology is as follows:

- First, we derive a one-dimensional system for the evolution of the half-saturation constant $k$ of the Monod kinetics (see (2.3)), based on the theory of adaptive dynamics.

- We then use this equation to determine the dilution rate optimizing the decrease of the trait $k$.

Assuming very small mutation rate, one may suppose that mutants appear sequentially. First, a monospecies population reaches its steady-state. Then, a first mutant - slightly different from the resident - appears. If it can invade the population, it becomes the new resident, and so on. This corresponds to the adaptive dynamics theory, widely used in ecology (see e.g $[10,11]$ ).

To model this phenomenon, we proceed as follows. Let us consider the half-saturation constant $k$ as the evolutionary trait of the resident. The dependency of the kinetics w.r.t. $k$ is now made explicit in the growth function: instead of considering an index $j$ for $\mu_{j}$ as in section 2 , we write the growth rate function as follows:

$$
\tilde{\mu}(s, k):=\bar{\mu} \frac{s}{k+s} .
$$

The dynamics of the resident is given by

$$
\left\{\begin{array}{l}
\dot{x}=\tilde{\mu}(s, k) x-u x \\
\dot{s}=-\tilde{\mu}(s, k) x+u\left(s_{i n}-s\right)
\end{array}\right.
$$

supposing the presence of only one species. Considering a constant dilution rate $u(t)=D<\tilde{\mu}\left(s_{i n}, k\right)$ for $t \geq 0$, the resident will reach the equilibrium point $\left(s_{i n}-\bar{s}, \bar{s}\right)$, where $\bar{s}=\frac{D k}{\bar{\mu}-D}$ (see $\left.e . g[23]\right)$. In this condition, the invasion fitness of a mutant - assuming that it is rare - is given by

$$
f\left(k, k^{\prime}\right)=\tilde{\mu}\left(\bar{s}, k^{\prime}\right)-D=\tilde{\mu}\left(\frac{D k}{\bar{\mu}-D}, k^{\prime}\right)-D
$$

where $k^{\prime}$ denotes the trait of the mutant, slightly different from the trait of the resident $k$. From the selection gradient, one can derive the evolution of the trait $k$ which is given by the ordinary differential equation (see e.g $[10,11])$ :

$$
\dot{k}=m(k) \frac{\partial f}{\partial k^{\prime}}\left(k, k^{\prime}\right)_{\left.\right|_{k^{\prime}=k}}=m(k) \frac{\partial \tilde{\mu}}{\partial k^{\prime}}\left(\bar{s}, k^{\prime}\right)_{\left.\right|_{k^{\prime}=k}}=-m(k) \bar{\mu} \frac{\bar{s}}{(k+\bar{s})^{2}}=-\frac{m(k)}{k \bar{\mu}} D(\bar{\mu}-D),
$$

where $m(k)>0$ is related to the mutation rate. Note that a similar equation can be obtained with a structured model such as system (2.2) in the limit of small mutation rate, when the population concentrates on a dirac mass (see e.g $[19,12])$.

In order to speed up the mutation-selection process, one should maximize $-\dot{k}$ (recalling that we are interested in the lowest $k$ in order to reach the target $\mathcal{T}$ in minimal time). Now, for a given value of the parameter $k$, the problem amounts to finding an optimal dilution rate maximizing the function

$$
D \longmapsto \psi(D):=\frac{m(k)}{k \bar{\mu}} D(\bar{\mu}-D) .
$$

We easily check that the maximum of $\psi$ is achieved for $D=\frac{\bar{\mu}}{2}$. Hence, in our setting, the dilution rate $D=\frac{\bar{\mu}}{2}$ should always be used to speed-up the selection, whatever is the trait $k$ of the resident.

Definition 4.1. The near optimal strategy is defined as the constant control $u=\frac{\bar{\mu}}{2}$. 
Remark 4.1. With $D=\frac{\bar{\mu}}{2}$, we get $\bar{s}=k$, i.e. the substrate concentration at equilibrium is equal to the half-saturation constant of the resident. Assuming small mutations (i.e. $k \simeq k^{\prime}$ ), this corresponds actually to the concentration that maximizes the difference between the growth rates of the mutant and the resident

$$
\Delta \mu_{k, k^{\prime}}(s):=\tilde{\mu}\left(s, k^{\prime}\right)-\tilde{\mu}(s, k)
$$

(indeed, a simple computation shows that $s \longmapsto \Delta \mu_{k, k^{\prime}}(s)$ is maximal for $s=\sqrt{k k^{\prime}} \simeq k$ ). This is in line with the results of [1] concerning the selection with two species in competition: we have shown that the singular strategy which regulates the concentration at a set-point corresponding to the maximum of the difference between the growth rate of both species is optimal in this case (see [1]).
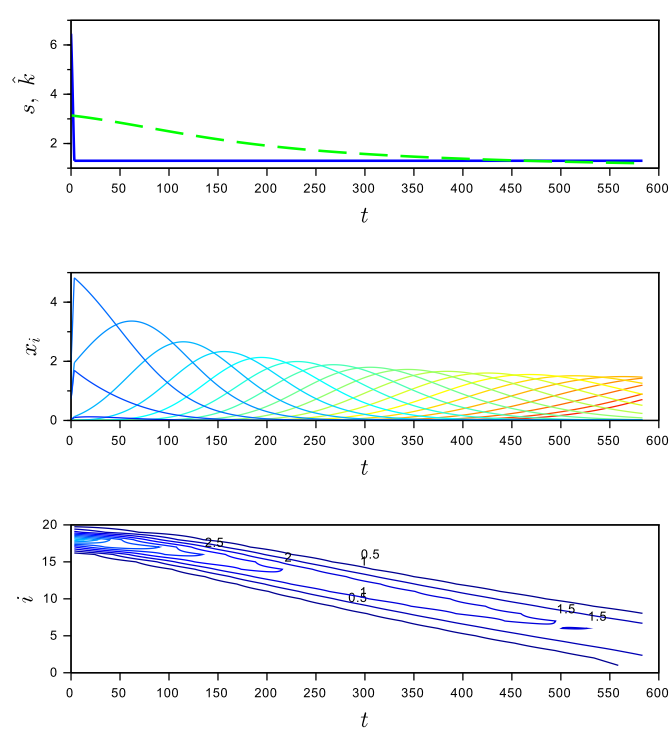
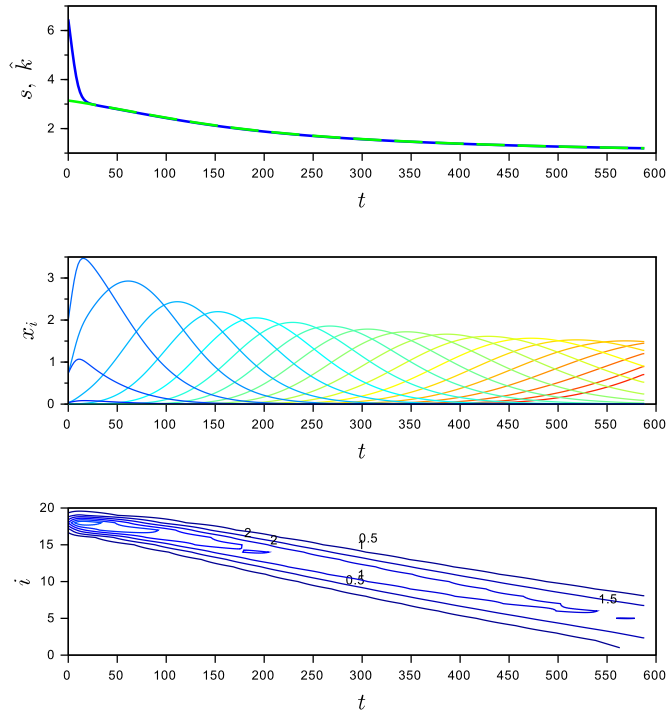

Figure 4: Comparison of the optimal strategy, i.e. an auxostat given by Control (3.19) (left) and the nearoptimal strategy, i.e. a chemostat with a dilution rate $D=\bar{\mu} / 2$ (right). Picture Top: Time evolution of the substrate concentration $s$ (blue line), and weighted averaged half-saturation constant $\hat{k}$ (green dashed line). Picture middle: time-evolution of biomass concentration $x_{i}$. Picture down: time-evolution of the distribution of species $i$.

We now investigate how the near-optimal strategy performs in comparison with the optimal strategy. Simulations have been carried out to evaluate this strategy using the original system $(2.6)$ with $n=20$ species (see Figure 4$)$. The target has been reached in $588 \mathrm{~h}$, which corresponds to a small increase $(+1 \%)$ in comparison to the optimal strategy $(582 \mathrm{~h})$ where the substrate concentration is regulated at $\sigma=1.35$. Thus, using $D=\bar{\mu} / 2$ is a strategy with near-optimal performance while being easily implementable. In this case, the substrate concentration follows the weighted averaged half-saturation constant $\hat{k}$, which facilitates the emergence of a mutant with a slightly lower half-saturation constant (see Remark 4.1).

Finally, we compute numerically the time to reach target using either control law (3.19) with different values of $\sigma$ (auxostat), or a constant dilution rate (chemostat), see Fig. 5. The performance of the auxostat strongly depends on $\sigma$, but one cannot determine the optimal value in practice (given that the system is unknown). In chemostat mode, the optimal value (for the constant dilution rate) is also dependent of the unknown system. Nonetheless, the near-optimal strategy, corresponding to $D=\bar{\mu} / 2=0.25$, is very close to the optimal value. The real strength of this strategy is that it does not require any knowledge on the system.

Selection experiments are generally carried out maintaining a low substrate concentration in an auxostat, or similarly a low dilution rate in a chemostat in order to exert a strong selection pressure. For example, in a selection experiment with the yeast Saccharomyces cerevisiae, Jansen et al. [16] used a dilution rate of $0.1 \mathrm{~h}^{-1}$, corresponding to approximately a quarter of the maximum growth rate of this species. In our setting, this value can actually slow down considerably the selection process in comparison with the optimal and near-optimal strategies. To conclude, the selection process is actually more rapid with a moderate selection 
pressure. Future work will explore whether this result still holds with more realistic model of selection-mutation (including stochastic effects such as genetic drifts [13]).
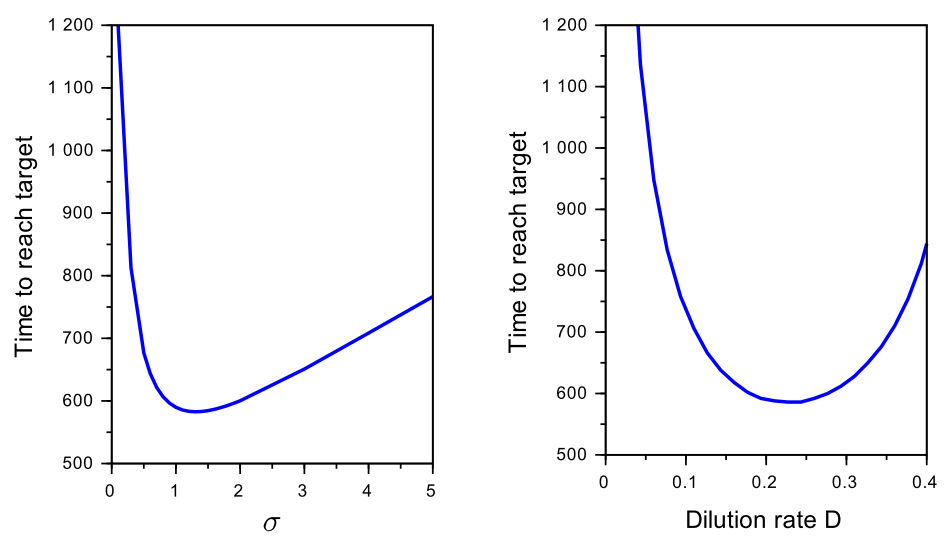

Figure 5: Time to reach the target as a function of $\sigma$ in auxostat (3.19) (left), or as a function of the dilution rate in chemostat (right).

\section{Conclusion}

Thanks to the Pontryagin Maximum Principle, we have proved that singular arcs of the problem (2.9) correspond to a constant value of the substrate concentration which depends on initial condition when $n \geq 3$. Moreover, an optimal feeding strategy is a concatenation of bang arcs and singular arcs which allows to provide an alternative to the competitive exclusion principle with a constant control. In order to optimize the species of interest, we believe that the optimal synthesis consists of a most rapid approach to a singular arc with at most one switching point. When $n=2$, we know that the proposed feedback control is optimal and we conjecture that this property remains valid whenever $n \geq 3$. This operating mode corresponds to an auxostat, which is commonly used for strain selection. The remaining difficulty for a real implementation is to determine the substrate level of the singular arc given that the species characteristics are unknown. To overcome this obstacle, we have defined a near-optimal strategy that can be easily implemented and that relies on the constant control $u=\frac{\bar{\mu}}{2}$. This value appears to be a good choice from the adaptive dynamics point of view.

\section{Acknowledgments}

This research benefited from the support of the "FMJH Program Gaspard Monge in optimization and operation research", from the support to this program from EDF. The authors are grateful to A. Rapaport and O. Bernard for helful discussions on the subject.

\section{References}

[1] T. Bayen, F. Mairet, Optimization of the separation of two species in a chemostat, Automatica (2014), Vol. 50, 4, 2014, pp. 1243-1248.

[2] W.N. Bennett, M.E. BoraAs, Isolation of a fast-growing strain of the rotifer Brachionus calyciflorus Pallas using turbidostat culture, Aquaculture, Vol. 73, pp. 27-36, 1988.

[3] J.-F. Bonnans, V. Grelard, P. Martinon, Bocop, the optimal control solver, Open source toolbox for optimal control problems, http://bocop.org, 2011.

[4] B. Bonnard, M. Chyba, Singular Trajectories and their role in Control Theorey, Springer, SMAI, vol. 40, 2002. 
[5] U. Boscain, B. Piccoli, Optimal Syntheses for Control Systems on 2-D Manifolds, Springer SMAI, vol. 43, 2004.

[6] M. Bardi, I. Capuzzo-Dolcetta, Optimal Control and Viscosity Solutions of Hamilton-JacobiBellman Equations, Birkhäuser, 1997.

[7] P. Cartigny, A. Rapaport, Turnpike theorem by a value approach function, ESAIM: COCV, 10, 123-141, 2004.

[8] L. Cesari, Optimization-Theory and Applications. Problems with ordinary differential equations, Springer, 1983.

[9] P. De Leenheer, J. Dockery, T. Gedeon, S. Pilyugin, The chemostat with lateral gene transfer, Journal of biological dynamics, Vol. 4, 6, pp. 607-620, 2010.

[10] U. Dieckmann, R. LAW, The dynamical theory of coevolution: a derivation from stochastic ecological processes, Journal of mathematical biology, Vol. 34, pp. 579-612, 1996.

[11] O. Diekmann, A beginners guide to adaptive dynamics, Banach Center publications, Vol. 63, pp. 47-86, 2004.

[12] O. Diekmann, P.-E. Jabin, S. Mischler, B. Perthame, The dynamics of adaptation: an illuminating example and a Hamilton-Jacobi approach, Theoretical population biology, Vol. 67, pp. 257-271,2005.

[13] S. ElenA AND R. Lenski, Evolution experiments with microorganisms: the dynamics and genetic bases of adaptation, Nature Reviews Genetics, Vol. 4, 6, pp. 457-469, 2003.

[14] P. Gajardo, H. Ramirez, A. Rapaport, Minimal time sequential batch reactors with bounded and impulse controls for one or more species, SIAM J. Control Optim., Vol. 47, 6, pp. 2827-2856, 2008.

[15] W. Harder, J.G. Kuenen, A. Matin, A review. Microbial selection in continuous culture, J. Appl. Bacteriol, Vol. 43, 1, pp. 2827-2856, 1977.

[16] M. Jansen, J. Diderich, M. Mashego, A. Hassane, J. De Winde, P. Daran-Lapujade, And J. PRONK, Prolonged selection in aerobic, glucose-limited chemostat cultures of Saccharomyces cerevisiae causes a partial loss of glycolytic capacity, Microbiology, Vol. 151, 5, pp. 1657-1669, 2005.

[17] H. Schattler, U. Ledzewicz, Geometric Optimal Control, Springer 2012.

[18] P. Masci, O. Bernard, F. Grognard, Continuous selection of the fastest growing species in the chemostat, In Proceedings of the 17th IFAC World Congress, Seoul, Korea, 2008.

[19] S. Mirrahimi, B. Perthame, J.Y. Wakano, Evolution of species trait through resource competition, Journal of mathematical biology, Vol. 64, 7, pp. 1189-1223, 2012.

[20] A. Novick, L. SzILARD, Experiments with the chemostat on spontaneous mutations of bacteria, PNAS 36: pp.708-719, 1950.

[21] B. Perthame, Transport Equations in Biology, Birkhäuser, 2007.

[22] L.S. Pontryagin, V.G. Boltyanskiy, R.V. Gamkrelidze, E.F. Mishchenko, Mathematical theory of optimal processes, The Macmillan Company, 1964.

[23] H.L. Smith and P. Waltman, The theory of the chemostat, Dynamics of microbial competition, Cambridge University Press, 1995.

[24] R. Vinter, Optimal Control, Systems and Control: Foundations and Applications, Birkhauser, 2000. 